\title{
Trapping region for discontinuous quasilinear elliptic systems of mixed monotone type
}

\author{
Siegfried Carl ${ }^{\mathrm{a}, 1}$ and Joseph W. Jerome ${ }^{\mathrm{b}}$

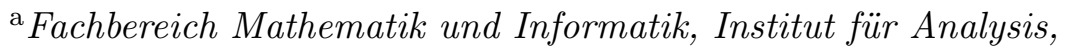 \\ Martin-Luther-Universität Halle-Wittenberg, \\ D-06099 Halle, Germany \\ ${ }^{\mathrm{b}}$ Department of Mathematics, Northwestern University, \\ Evanston, IL 60208, USA
}

\begin{abstract}
We consider discontinuous quasilinear elliptic systems with nonlinear boundary conditions of mixed Dirichlet-Robin type on the individual components. The system considered is of the general form $\mathcal{A} u+f(\cdot, u)=h$, where $\mathcal{A}$ is a quasilinear elliptic operator of Leray-Lions type, $u=\left(u_{1}, u_{2}\right)$, and the vector field $f=\left(f_{1}, f_{2}\right)$ is assumed to be of mixed monotone type associated with competitive or cooperative species. The vector field $f$ may be discontinuous with respect to all its arguments. The main goal is to prove the existence of solutions within the so-called trapping region. Furthermore, if, in addition, the components $f_{k}$ are continuous in their offdiagonal (nonprincipal) arguments, one can show the compactness of the solution set within the trapping region. The main tools used in the proof of our main result are variational inequalities, truncation and comparison techniques employing special test functions, and Tarski's fixed point theorem on complete lattices. Two applications of the theory developed in this paper are provided. The first application deals with the steady-state transport of two species of opposite charge within a physical channel, and in the second application a fluid medium is considered which may undergo a change of phase, and which acts as a carrier for certain solute species.
\end{abstract}

Key words: Quasilinear elliptic systems, discontinuous vector fields, systems of variational inequalities, Tarski fixed point theorem, competing and cooperating systems

1 current address: Florida Institute of Technology, Department of Mathematical Sciences, 150 West University Boulevard, Melbourne, Fl 32901-6988, U. S. A. 


\section{Introduction}

Let $\Omega \subset \mathbb{R}^{N}$ be a bounded domain with $C^{1}$-boundary $\partial \Omega$, and $\Gamma \subset \partial \Omega$ be such that $\partial \Omega \backslash \Gamma$ is a nonempty relatively open $C^{1}$-portion of $\partial \Omega$ (having positive surface measure). We consider the following elliptic system under mixed boundary conditions:

$$
\begin{array}{r}
A_{k} u_{k}+f_{k}\left(\cdot, u_{1}, u_{2}\right)=h_{k}, \text { in } \Omega, \\
u_{k}=0 \quad \text { on } \partial \Omega \backslash \Gamma, \quad \frac{\partial u_{k}}{\partial \nu_{k}}+g_{k}\left(\cdot, u_{k}\right)=0 \text { on } \Gamma,
\end{array}
$$

where $k=1,2$, and $A_{k}$ is a quasilinear elliptic operator of Leray-Lions type in the form

$$
A_{k} w(x)=-\sum_{i=1}^{N} \frac{\partial}{\partial x_{i}} a_{i}^{(k)}(x, \nabla w(x)),
$$

$\partial / \partial \nu_{k}$ denotes the outer conormal derivative on $\Gamma$ related to $A_{k}$. Since we allow each component $f_{k}: \Omega \times \mathbb{R} \times \mathbb{R} \rightarrow \mathbb{R}$ of the vector field $f=\left(f_{1}, f_{2}\right)$ to be discontinuous in all its arguments, the structure of the vector field $f$ is significant. We shall impose mixed monotonicity properties on $f$ that include models of cooperative and competing species.

In this paper we extend the results obtained by the second author in [8] and provide an alternative approach to deal with discontinuous systems. One of the goals of this paper is to provide a natural category of discontinuous systems of mixed monotone type for which solutions do occur in the so called trapping region formed by appropriately defined upper and lower solutions of (1.1). Moreover, we prove the compactness of the solution set enclosed by the trapping region when the components $f_{k}$ are, in addition, continuous in their nonprincipal arguments. By a principal argument we mean the argument $u_{k}$ of $f_{k}$. Applications of the theory developed here will be given.

While for continuous vector fields of Carathéodory type, even without any monotonicity conditions, this kind of trapping principle is well known (see, e.g., $[2,10])$, things become much more involved in case of discontinuous vector fields. Semilinear discontinuous systems in the form $\mathcal{A} u=f(\cdot, u)$ with $\mathcal{A} u=$ $\left(A_{1} u_{1}, \ldots, A_{N} u_{N}\right), f=\left(f_{1}, \ldots, f_{N}\right)$ and $u=\left(u_{1}, \ldots, u_{N}\right)$ have been treated in $[4,11]$, and the existence of solutions within the trapping region of upper and lower solutions has been proved provided that there is a vector field $M(u)=$ $\left(M_{1}\left(u_{1}\right), \ldots, M_{N}\left(u_{N}\right)\right)$ of increasing and continuous functions $M_{k}\left(u_{k}\right)$ such that the modified vector field $f(u)+M(u)$ is monotone increasing. The mixed monotonicity assumed in the present paper differs from that in the above cited papers $[4,11]$ and requires different tools. As will be seen later there are simple examples of discontinuous cooperative systems showing that there are no solutions at all in the conventional sense within the trapping region. Thus, it will be necessary for us to interpret 'solution' of (1.1) as solution of an 
associated multivalued system, whose precise definition will be given in the next section.

The primary tools used in the proof of our main result are variational inequalities, truncation and comparison techniques employing special test functions, and Tarski's fixed point theorem on complete lattices. The paper is organized as follows: In section 2 we define the trapping region and introduce an appropriate notion of solution of the problem under consideration motivated by a simple example. In section 3 we provide some tools, which will be used to prove our main result in section 4 . Relevant applications will be given in section 5 to demonstrate the applicability of the obtained results.

\section{Notations and Hypotheses}

Let $V:=W^{1, p}(\Omega)$ denote the usual (real) Sobolev space with $1<p<\infty$, and let $V_{0} \subset V$ be the subspace of $V$ defined by

$$
V_{0}=\{u \in V \mid \gamma u=0 \text { on } \partial \Omega \backslash \Gamma\}
$$

where $\gamma: V \rightarrow L^{p}(\partial \Omega)$ is the trace operator which is linear and compact, cf., e.g., [9]. (In what follows we keep the notation $\gamma$ also for its restriction to $\Gamma$.)

We introduce the natural partial ordering in $L^{p}(\Omega)$, that is $u \leq w$ if and only if $w-u$ belongs to the positive cone $L_{+}^{p}(\Omega)$ of all nonnegative elements of $L^{p}(\Omega)$, which induces also a partial ordering in the Sobolev space $V$. If $u, w \in V$ and $u \leq w$ then

$$
[u, w]=\{v \in V \mid u \leq v \leq w\}
$$

denotes the order interval formed by $u$ and $w$.

We assume $h_{k} \in V^{*}$, where $V^{*}$ is the dual space of $V$, and suppose for the data of the boundary value problem (1.1) the following regularity and growth conditions:

(A1) The coefficients $a_{i}^{(k)}: \Omega \times \mathbb{R}^{N} \rightarrow \mathbb{R}, i=1, \ldots, N$ are Carathéodory functions, i.e. each $a_{i}^{(k)}(x, \xi)$ is measurable in $x \in \Omega$ for all $\xi \in \mathbb{R}^{N}$ and continuous in $\xi \in \mathbb{R}^{N}$ for a.e. $x \in \Omega$. There is a constant $c_{1}>0$ and a function $k_{1} \in L_{+}^{q}(\Omega)$ with $q$ being the conjugate real, i.e., $1 / p+1 / q=1$, such that for $k=1,2$,

$$
\left|a_{i}^{(k)}(x, \xi)\right| \leq k_{1}(x)+c_{1}|\xi|^{p-1}
$$

for a.e. $x \in \Omega$ and for all $\xi \in \mathbb{R}^{N}$. 
(A2)

$$
\sum_{i=1}^{N}\left(a_{i}^{(k)}(x, \xi)-a_{i}^{(k)}\left(x, \xi^{\prime}\right)\right)\left(\xi_{i}-\xi_{i}^{\prime}\right) \geq \mu\left|\xi-\xi^{\prime}\right|^{p}, \quad k=1,2,
$$

for a.e. $x \in \Omega$ and for all $\xi, \xi^{\prime} \in \mathbb{R}^{N}$ with $\mu$ being some positive constant.

(H1) The components $f_{k}: \Omega \times \mathbb{R} \times \mathbb{R} \rightarrow \mathbb{R}$ of the vector field $f=\left(f_{1}, f_{2}\right)$ are required to be Baire-measurable, and there is a function $k_{2} \in L_{+}^{q}(\Omega)$ such that

$$
\left|f_{k}(x, r, s)\right| \leq k_{2}(x), \quad k=1,2,
$$

for a.e. $x \in \Omega$ and $(r, s) \in\left[\left(w_{1}(x), w_{2}(x)\right),\left(v_{1}(x), v_{2}(x)\right)\right]$, where

$\left[\left(w_{1}, w_{2}\right),\left(v_{1}, v_{2}\right)\right]$ denotes some order interval in $L^{p}(\Omega) \times L^{p}(\Omega)$ with componentwise ordering, i.e., $\left(w_{1}, w_{2}\right) \leq\left(v_{1}, v_{2}\right)$ iff $w_{1} \leq v_{1}$ and $w_{2} \leq v_{2}$.

(H2) The nonlinearities of the boundary conditions $g_{k}: \Gamma \times \mathbb{R} \rightarrow \mathbb{R}$ are Carathéodory functions, and there is some $k_{3} \in L_{+}^{q}(\Gamma)$ such that

$$
\left|g_{k}(x, s)\right| \leq k_{3}(x), \quad k=1,2,
$$

for a.e. $x \in \Gamma$ and for all $s \in\left[\gamma w_{k}(x), \gamma v_{k}(x)\right]$ with $\left[w_{k}, v_{k}\right] \in V$ being some order interval. Moreover, $s \mapsto g_{k}(x, s)$ is increasing for a.e. $x \in \Gamma$.

Remark 2.1. According to hypothesis (H1) the components $f_{k}$ of the vector field $f$ may be discontinuous in all their arguments, and are required to satisfy only a local $L^{q}(\Omega)$-boundedness condition within some interval, which is weaker than the usual growth condition in the form $\left|f_{k}(x, r, s)\right| \leq$ $k_{2}(x)+c\left(|r|^{p-1}+|s|^{p-1}\right)$. The summation exponent of the bound $k_{2}$ can be improved by means of the Sobolev embedding theorem, which, however, has been omitted. The Baire-measurability of the components $f_{k}$, in particular, implies that the $f_{k}$ are superpositionally measurable, i.e., whenever $u, v: \Omega \rightarrow \mathbb{R}$ are measurable then also the superposition $x \mapsto f_{k}(x, u(x), v(x))$ is a measurable function in $\Omega$. By hypothesis (H2) we impose a boundedness only within some interval. The intervals for both (H1) and (H2) will be specified later. Homogeneous Dirichlet conditions have been assumed without loss of generality, since nonhomogeneous Dirichlet data can be reduced to homogeneous ones by simple translation without changing the structure of the BVP (1.1).

Let $a^{(k)}$ denote the semilinear form associated with the differential operator $A_{k}$ by

$$
\left\langle A_{k} u, \varphi\right\rangle:=a^{(k)}(u, \varphi)=\int_{\Omega} \sum_{i=1}^{N} a_{i}^{(k)}(x, \nabla u) \frac{\partial \varphi}{\partial x_{i}} d x, \quad \forall \varphi \in V_{0}
$$

Then by hypotheses (A1) the operator $A_{k}: V \rightarrow V_{0}^{*}$ is continuous and bounded, and by (A2) the operator $A_{k}: V_{0} \rightarrow V_{0}^{*}$ is uniformly monotone, 
since in $V_{0}$ an equivalent norm is given by

$$
\|u\|_{V_{0}}^{p}=\int_{\Omega}|\nabla u|^{p} d x
$$

Mixed monotone structure of the vector field. We distinguish two classes of vector fields corresponding to models of competing and cooperating species.

Competing Species: $f_{1}\left(\cdot, u_{1}, u_{2}\right)$ and $f_{2}\left(\cdot, u_{1}, u_{2}\right)$ are both separately increasing in $u_{1}, u_{2}$.

Cooperating Species: $f_{1}\left(\cdot, u_{1}, u_{2}\right)$ is increasing in $u_{1}$, and decreasing in $u_{2}$; $f_{2}\left(\cdot, u_{1}, u_{2}\right)$ is decreasing in $u_{1}$, and increasing in $u_{2}$.

Definition 2.1 Let $\bar{u}=\left(\bar{u}_{1}, \bar{u}_{2}\right)$ and $\underline{u}=\left(\underline{u}_{1}, \underline{u}_{2}\right)$ be from $V \times V$ such that $\underline{u} \leq \bar{u}$. The interval $[\underline{u}, \bar{u}] \subset V \times V$ is called a trapping region for the BVP (1.1) if (2.1) and (2.2) are satisfied with respect to $[\underline{u}, \bar{u}]$ and $\left[\underline{u}_{k}, \bar{u}_{k}\right] k=1,2$, respectively, and if the following inequalities hold:

$$
\begin{array}{r}
A_{1} \bar{u}_{1}+f_{1}\left(\cdot, \bar{u}_{1}, v\right) \geq h_{1}, \quad \forall v \in\left[\underline{u}_{2}, \bar{u}_{2}\right], \\
A_{2} \bar{u}_{2}+f_{2}\left(\cdot, v, \bar{u}_{2}\right) \geq h_{2}, \quad \forall v \in\left[\underline{u}_{1}, \bar{u}_{1}\right], \\
\bar{u}_{k} \geq 0 \quad \text { on } \partial \Omega \backslash \Gamma, \quad \frac{\partial \bar{u}_{k}}{\partial \nu_{k}}+g\left(\cdot, \bar{u}_{k}\right) \geq 0 \text { on } \Gamma, \\
A_{1} \underline{u}_{1}+f_{1}\left(\cdot, \underline{u}_{1}, v\right) \leq h_{1}, \quad \forall v \in\left[\underline{u}_{2}, \bar{u}_{2}\right], \\
A_{2} \underline{u}_{2}+f_{2}\left(\cdot, v, \underline{u}_{2}\right) \leq h_{2}, \quad \forall v \in\left[\underline{u}_{1}, \bar{u}_{1}\right], \\
\underline{u}_{k} \leq 0 \text { on } \partial \Omega \backslash \Gamma, \quad \frac{\partial \underline{u}_{k}}{\partial \nu_{k}}+g\left(\cdot, \underline{u}_{k}\right) \leq 0 \text { on } \Gamma .
\end{array}
$$

Remark 2.2. The inequalities (2.3) and (2.4) have to be understood in a weak sense, i.e., as inequalities with respect to the dual order cone in $V_{0}^{*}$, which for example for the component $\bar{u}_{1}$ of (2.3) reads as follows: $\gamma \bar{u}_{1} \geq 0$ on $\partial \Omega \backslash \Gamma$ and

$$
\begin{gathered}
a^{(1)}\left(\bar{u}_{1}, \varphi\right)+\int_{\Gamma} g_{1}\left(\cdot, \gamma \bar{u}_{1}\right) \gamma \varphi d \Gamma+\int_{\Omega} f_{1}\left(\cdot, \bar{u}_{1}, v\right) \varphi d x \geq\left\langle h_{1}, \varphi\right\rangle, \\
\forall v \in\left[\underline{u}_{2}, \bar{u}_{2}\right], \forall \varphi \in V_{0} \cap L_{+}^{p}(\Omega) .
\end{gathered}
$$

In the case that $h_{k}=0, g_{k}(\cdot, s)=0$ and $A_{k}$ are second order linear elliptic operators in divergence form, inequalities (2.3) and (2.4) correspond with the property of an outward pointing vector field on the boundary of a rectangle $Q$ formed by constant vectors $\underline{u}, \bar{u} \in \mathbb{R}^{2}$, cf. [8].

Our main goal is to show that under hypotheses (A1), (A2), (H1) and (H2) each trapping region for (1.1) contains al least one solution of the BVP (1.1). 
The following example provides some motivation for what is meant by a solution of the BVP (1.1).

Example. Let $p=2, A_{k}=-\Delta, h_{k}=0$, and $g_{k}(\cdot, s)=0$, and consider the simple system $(k=1,2)$

$$
\begin{gathered}
\Delta u_{k}=f_{i}\left(u_{1}, u_{2}\right), \quad \text { in } \Omega \\
u_{k}=0 \quad \text { on } \partial \Omega \backslash \Gamma, \quad \frac{\partial u_{k}}{\partial n}=0 \text { on } \Gamma,
\end{gathered}
$$

where the vector field $f=\left(f_{1}, f_{2}\right)$ is given by

$$
\begin{gathered}
f_{1}\left(u_{1}, u_{2}\right)=\left\{\begin{array}{ccc}
-1 & \text { if } & u_{1} \leq 0 \\
0 & \text { if } & u_{1}>0
\end{array}\right. \\
f_{2}\left(u_{1}, u_{2}\right)=0 .
\end{gathered}
$$

One readily verifies that the given vector field is a model of cooperative species, and that $[\underline{u}, \bar{u}]$ with $\underline{u}=(0,0)$ and $\bar{u}=(b, b)$ for any fixed $b>0$ forms a trapping region according to Definition 2.1; or, in other words, the property of an outward pointing vector field on the boundary of the rectangle $Q=$ $[0, b] \times[0, b]$ is satisfied. However, there are no solutions in the conventional sense within the trapping region, and, moreover, there are no solutions at all. This can be seen as follows. Our proof is by contradiction. Assume there would be a solution; then from the second equation together with the boundary conditions we infer that $u_{2}=0$ is the uniquely defined solution component. When this is substituted into the first equation, we see that $u_{1}$ has to satisfy the following boundary value problem:

$$
\Delta u_{1}=f_{1}\left(u_{1}, 0\right) \text { in } \Omega, \quad u_{1}=0 \quad \text { on } \partial \Omega \backslash \Gamma, \quad \frac{\partial u_{1}}{\partial n}=0 \text { on } \Gamma,
$$

whose weak formulation reads as

$$
\int_{\Omega}\left(\nabla u_{1} \nabla \varphi+f_{1}\left(u_{1}, 0\right) \varphi\right) d x=0, \quad \forall \varphi \in V_{0} .
$$

Taking in (2.8) the special test function $\varphi=u_{1}$, we obtain

$$
\int_{\Omega}\left(\nabla u_{1}\right)^{2} d x+\int_{\Omega} f_{1}\left(u_{1}, 0\right) u_{1} d x=0 .
$$

Since the second integral in (2.9) is nonnegative we obtain

$$
\int_{\Omega}\left(\nabla u_{1}\right)^{2} d x \leq 0
$$

which implies $u_{1}=0$. However $\left(u_{1}, u_{2}\right)=(0,0)$ is not a solution of $(2.5),(2.6)$; thus we arrive at a contradiction. 
This shows that the notion of solution must be extended. For this purpose we introduce the following multivalued vector field $\beta=\left(\beta_{1}, \beta_{2}\right)$, which is associated with the given vector field $f=\left(f_{1}, f_{2}\right)$ by:

$$
\begin{aligned}
& \beta_{1}\left(\cdot, u_{1}, u_{2}\right)=\left[f_{1}\left(\cdot, u_{1}-0, u_{2}\right), f_{1}\left(\cdot, u_{1}+0, u_{2}\right)\right], \\
& \beta_{2}\left(\cdot, u_{1}, u_{2}\right)=\left[f_{2}\left(\cdot, u_{1}, u_{2}-0\right), f_{2}\left(\cdot, u_{1}, u_{2}+0\right)\right],
\end{aligned}
$$

where $f_{1}\left(\cdot, u_{1} \pm 0, u_{2}\right)$ and $f_{2}\left(\cdot, u_{1}, u_{2} \pm 0\right)$ denote the one-sided limits of $f_{1}$ and $f_{2}$ with respect to the principal argument. Thus for both models $\beta_{k}$ : $\Omega \times \mathbb{R} \times \mathbb{R} \rightarrow 2^{\mathbb{R}} \backslash \emptyset$ is a maximal monotone graph with respect to its principal argument. Now by a solution of the BVP (1.1) we mean a solution of the following associated system of elliptic inclusions: $(k=1,2)$

$$
\begin{array}{r}
A_{k} u_{k}+\beta_{k}\left(\cdot, u_{1}, u_{2}\right) \ni h_{k}, \text { in } \Omega, \\
u_{k}=0 \quad \text { on } \partial \Omega \backslash \Gamma, \quad \frac{\partial u_{k}}{\partial \nu_{k}}+g_{k}\left(\cdot, u_{k}\right)=0 \text { on } \Gamma .
\end{array}
$$

The weak formulation of the multivalued system (2.11) is given by the next definition.

Definition 2.2. The couple of pairs $\left\{\left(u_{1}, w_{1}\right),\left(u_{2}, w_{2}\right)\right\} \in\left(V_{0} \times L^{q}(\Omega)\right) \times\left(V_{0} \times\right.$ $L^{q}(\Omega)$ ) is called a solution of the system (2.11) if for $k=1,2$, the following is fulfilled:

(i) $w_{k}(x) \in \beta_{k}\left(x, u_{1}(x), u_{2}(x)\right)$ for a.e. $x \in \Omega$;

(ii) $a^{(k)}\left(u_{k}, \varphi\right)+\int_{\Gamma} g_{k}\left(\cdot, \gamma u_{k}\right) \gamma \varphi d \Gamma+\int_{\Omega} w_{k} \varphi d x=\left\langle h_{k}, \varphi\right\rangle, \quad \forall \varphi \in V_{0}$.

Remark 2.3. In the sense of Definition 2.2 the above example possesses the unique solution $\left\{\left(u_{1}, w_{1}\right),\left(u_{2}, w_{2}\right)\right\}=\{(0,0),(0,0)\}$.

Our main goal is to provide existence and compactness results within the trapping region for the multivalued version (2.11) of the BVP (1.1). Before proving our main result we provide in the next section some preliminaries.

\section{Preliminaries}

Let $[\underline{u}, \bar{u}] \subset V \times V$ be a trapping region for the BVP (1.1) according to Definition 2.1, and let $\alpha>0$ be any given constant. Assume that (2.1) of (H1) is satisfied with respect to the order interval $[\underline{u}-(\alpha, \alpha), \bar{u}+(\alpha, \alpha)]$, and (H2) with respect to $\left[\underline{u}_{k}, \bar{u}_{k}\right], k=1,2$, where $\underline{u}_{k}$ and $\bar{u}_{k}$ are the components of $\underline{u}$ and $\bar{u}$ of the trapping interval. Also hypotheses (A1) and (A2) are assumed throughout this section. For $\eta=0, \eta=\alpha$, and $k=1,2$, we introduce truncation operators 
$T_{k}^{\eta}$ by

$$
T_{k}^{\eta} u(x)=\left\{\begin{array}{rll}
\bar{u}_{k}(x)+\eta & \text { if } & u(x)>\bar{u}_{k}(x)+\eta, \\
u(x) & \text { if } \quad & \underline{u}_{k}(x)-\eta \leq u(x) \leq \bar{u}_{k}(x)+\eta, \\
\underline{u}_{k}(x)-\eta & \text { if } & u(x)<\underline{u}_{k}(x)-\eta .
\end{array}\right.
$$

It is well known that $T_{k}^{\eta}: V \rightarrow V$ are continuous and bounded, cf., e.g., [3,6]. By means of $T_{k}^{\eta}$ we define a new vector field which is related to $f=\left(f_{1}, f_{2}\right)$ by

$$
\begin{aligned}
f_{1}^{\alpha}\left(x, u_{1}(x), u_{2}(x)\right) & :=f_{1}\left(x, T_{1}^{\alpha} u_{1}(x), u_{2}(x)\right), \\
f_{2}^{\alpha}\left(x, u_{1}(x), u_{2}(x)\right) & :=f_{2}\left(x, u_{1}(x), T_{2}^{\alpha} u_{2}(x)\right),
\end{aligned}
$$

and consider the following functionals

$$
\begin{aligned}
& J_{1}\left(u_{1}, u_{2}\right):=\int_{\Omega}\left(\int_{0}^{u_{1}(x)} f_{1}^{\alpha}\left(x, s, u_{2}(x)\right) d s\right) d x \\
& J_{2}\left(u_{1}, u_{2}\right):=\int_{\Omega}\left(\int_{0}^{u_{2}(x)} f_{2}^{\alpha}\left(x, u_{1}(x), s\right) d s\right) d x .
\end{aligned}
$$

Lemma 3.1. In either case of the two classes of vector fields $f$ we have that for any $v_{2} \in\left[\underline{u}_{2}, \bar{u}_{2}\right]$ the functional $J_{1}\left(\cdot, v_{2}\right): L^{p}(\Omega) \rightarrow \mathbb{R}$ is Lipschitz continuous, convex, and the subdifferential of $J_{1}$ with respect to its principal argument is given by

$$
\partial_{1} J_{1}\left(u_{1}, v_{2}\right)(x)=\left[f_{1}^{\alpha}\left(x, u_{1}(x)-0, v_{2}(x)\right), f_{1}^{\alpha}\left(x, u_{1}(x)+0, v_{2}(x)\right)\right] \subset L^{q}(\Omega),
$$

which holds in $L^{p}(\Omega)$ and in $V$ as well. Analogously, for any $v_{1} \in\left[\underline{u}_{1}, \bar{u}_{1}\right]$, the functional $J_{2}\left(v_{1}, \cdot\right): L^{p}(\Omega) \rightarrow \mathbb{R}$ is Lipschitz continuous, convex, and the subdifferential with respect to its principal argument is given by

$$
\partial_{2} J_{2}\left(v_{1}, u_{2}\right)(x)=\left[f_{2}^{\alpha}\left(x, v_{1}(x), u_{2}(x)-0\right), f_{2}^{\alpha}\left(x, v_{1}(x), u_{2}(x)+0\right)\right] \subset L^{q}(\Omega) .
$$

Proof. By hypothesis (H1) and the definition of the truncations the function $(x, s) \mapsto f_{1}^{\alpha}\left(x, s, v_{2}(x)\right)$ is Baire-measurable and dominated by $k_{2} \in L_{+}^{q}(\Omega)$, which yields

$$
\left|J_{1}\left(u_{1}, v_{2}\right)-J_{1}\left(\hat{u}_{1}, v_{2}\right)\right| \leq \int_{\Omega} k_{2}(x)\left|u_{1}(x)-\hat{u}_{1}(x)\right| d x \leq\left\|k_{2}\right\|_{L^{q}(\Omega)}\left\|u_{1}-\hat{u}_{1}\right\|_{L^{p}(\Omega)},
$$

i.e., Lipschitz continuity. Furthermore, since $s \mapsto f_{1}^{\alpha}\left(x, s, v_{2}(x)\right)$ is increasing for both classes of vector fields, the functional $J_{1}\left(\cdot, v_{2}\right): L^{p}(\Omega) \rightarrow \mathbb{R}$ is convex. Thus, due to Chang [5, Theorem 2.3] the subdifferential $\partial_{1} J_{1}\left(u_{1}, v_{2}\right)$ is given by (3.3). 
We associate with the system (2.11) of inclusions the following 'truncated' system of variational inequalities: Find $u=\left(u_{1}, u_{2}\right) \in V_{0} \times V_{0}$ such that for all $\varphi \in V_{0}$

$$
\begin{aligned}
a^{(1)}\left(u_{1}, \varphi-u_{1}\right) & +\int_{\Gamma} g_{1}\left(\cdot, T_{1}^{0} \gamma u_{1}\right)\left(\gamma \varphi-\gamma u_{1}\right) d \Gamma \\
& +J_{1}\left(\varphi, u_{2}\right)-J_{1}\left(u_{1}, u_{2}\right) \geq\left\langle h_{1}, \varphi-u_{1}\right\rangle, \\
a^{(2)}\left(u_{2}, \varphi-u_{2}\right) & +\int_{\Gamma} g_{2}\left(\cdot, T_{2}^{0} \gamma u_{2}\right)\left(\gamma \varphi-\gamma u_{2}\right) d \Gamma \\
& +J_{2}\left(u_{1}, \varphi\right)-J_{2}\left(u_{1}, u_{2}\right) \geq\left\langle h_{2}, \varphi-u_{2}\right\rangle
\end{aligned}
$$

where the truncations $T_{k}^{0}$ acting on the traces are defined in a similar way as for functions on $\Omega$. System (3.5) will play a crucial role in the proof of our main result.

Lemma 3.2. Let $[\underline{u}, \bar{u}] \subset V \times V$ be the given trapping region. Under the hypotheses of this section $u \in[\underline{u}, \bar{u}]$ is a solution of the system (2.11) if and only if $u$ is a solution of the system of variational inequalities (3.5).

Proof. Let $u \in[\underline{u}, \bar{u}]$ be a solution of (3.5). Then $T_{k}^{0} u_{k}=u_{k}$ for $k=1,2$. By hypothesis (H2) the operator $G_{k}: V_{0} \rightarrow V_{0}^{*}$ given by

$$
\left\langle G_{k} u_{k}, \varphi\right\rangle=\int_{\Gamma} g_{k}\left(\cdot, T_{k}^{0} \gamma u_{k}\right) \gamma \varphi d \Gamma, \forall \varphi \in V_{0}
$$

is well-defined (even continuous and bounded), and yields for $u \in[\underline{u}, \bar{u}]$

$$
\left\langle G_{k} u_{k}, \varphi\right\rangle=\int_{\Gamma} g_{k}\left(\cdot, \gamma u_{k}\right) \gamma \varphi d \Gamma
$$

From (3.5) we obtain

$$
-A_{k} u_{k}-G_{k} u_{k}+h_{k} \in \partial_{k} J_{k}\left(u_{1}, u_{2}\right) \text {, in } V_{0}^{*} \text {. }
$$

In view of Lemma 3.1 we have $\partial_{k} J_{k}\left(u_{1}, u_{2}\right) \subset L^{q}(\Omega) \subset V_{0}^{*}$, and thus by (3.6) there is a function $w_{k} \in L^{q}(\Omega)$ satisfying

$$
-A_{k} u_{k}-G_{k} u_{k}+h_{k}=w_{k} \text { in } V_{0}^{*} .
$$

Furthermore, by (3.3) and (3.4) we have

$$
\begin{aligned}
& w_{1}(x) \in\left[f_{1}^{\alpha}\left(x, u_{1}(x)-0, u_{2}(x)\right), f_{1}^{\alpha}\left(x, u_{1}(x)+0, u_{2}(x)\right)\right] \\
& =\beta_{1}\left(x, u_{1}(x), u_{2}(x)\right), \\
& w_{2}(x) \in\left[f_{2}^{\alpha}\left(x, u_{1}(x), u_{2}(x)-0\right), f_{2}^{\alpha}\left(x, u_{1}(x), u_{2}(x)+0\right)\right] \\
& =\beta_{2}\left(x, u_{1}(x), u_{2}(x)\right),
\end{aligned}
$$

where the equalities on the right-hand side of (3.8) are a consequence of $T_{k}^{\alpha}\left(u_{k} \pm \varepsilon\right)=u_{k} \pm \varepsilon$ for any $\varepsilon \in(0, \alpha)$. But (3.7) and (3.8) are nothing else than the weak formulation of (2.11) according to Definition 2.2. 
Conversely, let $u \in[\underline{u}, \bar{u}]$ be a solution of (2.11), which, according to Definition 2.2, means that there are $w_{k} \in L^{q}(\Omega), k=1,2$, such that $w_{k}(x) \in$ $\beta_{k}\left(x, u_{1}(x), u_{2}(x)\right)$ for a.e. $x \in \Omega$ and

$$
a^{(k)}\left(u_{k}, \varphi\right)+\int_{\Gamma} g_{k}\left(\cdot, \gamma u_{k}\right) \gamma \varphi d \Gamma+\int_{\Omega} w_{k} \varphi d x=\left\langle h_{k}, \varphi\right\rangle, \forall \varphi \in V_{0} .
$$

Since $u \in[\underline{u}, \bar{u}]$, it follows that its components $u_{1}$ and $u_{2}$ satisfy the identity

$$
\begin{aligned}
& \beta_{1}\left(x, u_{1}(x), u_{2}(x)\right)=\left[f_{1}^{\alpha}\left(x, u_{1}(x)-0, u_{2}(x)\right), f_{1}^{\alpha}\left(x, u_{1}(x)+0, u_{2}(x)\right)\right] \\
& \beta_{2}\left(x, u_{1}(x), u_{2}(x)\right)=\left[f_{2}^{\alpha}\left(x, u_{1}(x), u_{2}(x)-0\right), f_{2}^{\alpha}\left(x, u_{1}(x), u_{2}(x)+0\right)\right] .
\end{aligned}
$$

The function $(x, s) \mapsto f_{1}^{\alpha}\left(x, s, u_{2}(x)\right)$ is superpositionally measurable, increasing in $s$ and dominated by $k_{2} \in L^{q}(\Omega)$, which implies for a.e. $x \in \Omega$ the inequality

$$
w_{1}(x)\left(\varphi(x)-u_{1}(x)\right) \leq \int_{u_{1}(x)}^{\varphi(x)} f_{1}^{\alpha}\left(x, s, u_{2}(x)\right) d s, \forall \varphi \in L^{p}(\Omega),
$$

and thus integration over $\Omega$ yields

$$
\int_{\Omega} w_{1}\left(\varphi-u_{1}\right) d x \leq J_{1}\left(\varphi, u_{2}\right)-J_{1}\left(u_{1}, u_{2}\right) .
$$

The last inequality, in particular, holds for all $\varphi \in V_{0}$. Replacing $\varphi$ in (3.9) for $k=1$ by $\varphi-u_{1}$ and using (3.11) we obtain the first variational inequality of (3.5). Here we have used also the fact that $T_{1}^{0} u_{1}=u_{1}$ within the trapping region. The second variational inequality of (3.5) can be deduced in just the same way, which completes the proof.

The next lemma provides an existence and uniqueness result for the individual variational inequalities of (3.5) with respect to their principal argument while the other is fixed. More precisely we have the following result.

Lemma 3.3. Let $v_{2} \in\left[\underline{u}_{2}, \bar{u}_{2}\right]$ be fixed. Then the following variational inequality has a uniquely defined solution: Find $u_{1} \in V_{0}$ such that for all $\varphi \in V_{0}$

$$
\begin{aligned}
a^{(1)}\left(u_{1}, \varphi-u_{1}\right) & +\int_{\Gamma} g_{1}\left(\cdot, T_{1}^{0} \gamma u_{1}\right)\left(\gamma \varphi-\gamma u_{1}\right) d \Gamma \\
& +J_{1}\left(\varphi, v_{2}\right)-J_{1}\left(u_{1}, v_{2}\right) \geq\left\langle h_{1}, \varphi-u_{1}\right\rangle .
\end{aligned}
$$

Replace $u_{1}$ in the second variational inequality of (3.5) by any fixed $v_{1} \in$ $\left[\underline{u}_{1}, \bar{u}_{1}\right]$; then analogously there exists a unique solution $u_{2} \in V_{0}$ of

$$
\begin{aligned}
a^{(2)}\left(u_{2}, \varphi-u_{2}\right) & +\int_{\Gamma} g_{2}\left(\cdot, T_{2}^{0} \gamma u_{2}\right)\left(\gamma \varphi-\gamma u_{2}\right) d \Gamma \\
& +J_{2}\left(v_{1}, \varphi\right)-J_{2}\left(v_{1}, u_{2}\right) \geq\left\langle h_{2}, \varphi-u_{2}\right\rangle
\end{aligned}
$$


Proof. The proof will be given only for the variational inequality (3.12), since the proof for (3.13) follows in just the same way. By (A1) and (A2) the operator $A_{1}: V_{0} \rightarrow V_{0}^{*}$ that is associated with the semilinear form $a^{(1)}$ is continuous, bounded and uniformly monotone. By (H2) the operator $G_{1}: V_{0} \rightarrow V_{0}^{*}$ given by

$$
\left\langle G_{1} u_{1}, \varphi\right\rangle=\int_{\Gamma} g_{1}\left(\cdot, T_{1}^{0} \gamma u_{1}\right) \gamma \varphi d \Gamma, \forall \varphi \in V_{0},
$$

is continuous, bounded, and, in view of the monotonicity of $g_{1}(x, s)$ in $s$, also monotone. Thus the sum $A_{1}+G_{1}: V_{0} \rightarrow V_{0}^{*}$ is continuous, bounded, and uniformly monotone, which, in particular, implies the coercivity of $A_{1}+$ $G_{1}$. Since the functional $J_{1}\left(\cdot, v_{2}\right): V_{0} \rightarrow \mathbb{R}$ is convex and continuous (even Lipschitz continuous), the existence of a uniquely defined solution of (3.12) follows from [13, Theorem 54.A].

\section{Main results}

In this section we prove the existence of solutions within the trapping region of the system of elliptic inclusions (2.11) which is the multivalued version of the BVP (1.1). The two classes of models, the competing species and cooperating species, will be treated separately. One of the main tools in the proofs is Tarski's fixed point theorem, which we recall in Lemma 4.1, cf., e.g., [12, Theorem 11.E.].

Lemma 4.1. Every monotone increasing mapping $P: X \rightarrow X$ on a complete lattice $X$ has a smallest and a greatest fixed point.

Theorem 4.1. Let $f=\left(f_{1}, f_{2}\right)$ be the vector field corresponding to the competing species model. Assume (A1) and (A2), and let $[\underline{u}, \bar{u}] \subset V \times V$ be a trapping region for the BVP (1.1) according to Definition 2.1. Let $\alpha>$ 0 be any given constant so that (2.1) of (H1) is satisfied with respect to $[\underline{u}-(\alpha, \alpha), \bar{u}+(\alpha, \alpha)]$, and (H2) with respect to $\left[\underline{u}_{k}, \bar{u}_{k}\right], k=1,2$, where $\underline{u}_{k}$ and $\bar{u}_{k}$ are the components of $\underline{u}$ and $\bar{u}$ of the trapping interval. Then there exist solutions of the system (2.11) within $[\underline{u}, \bar{u}]$.

Proof. By means of Lemma 3.2 the existence of solutions for (2.11) is equivalent to the existence of solutions for the system of variational inequalities (3.5) within $[\underline{u}, \bar{u}]$. Its proof is mainly based on Tarski's fixed point theorem. To this end the system (3.5), which is given by the following two coupled variational inequalities for $\left(u_{1}, u_{2}\right) \in V_{0} \times V_{0}$,

$$
\begin{aligned}
a^{(1)}\left(u_{1}, \varphi-u_{1}\right) & +\int_{\Gamma} g_{1}\left(\cdot, T_{1}^{0} \gamma u_{1}\right)\left(\gamma \varphi-\gamma u_{1}\right) d \Gamma \\
& +J_{1}\left(\varphi, u_{2}\right)-J_{1}\left(u_{1}, u_{2}\right) \geq\left\langle h_{1}, \varphi-u_{1}\right\rangle, \forall \varphi \in V_{0}
\end{aligned}
$$




$$
\begin{aligned}
a^{(2)}\left(u_{2}, \varphi-u_{2}\right) & +\int_{\Gamma} g_{2}\left(\cdot, T_{2}^{0} \gamma u_{2}\right)\left(\gamma \varphi-\gamma u_{2}\right) d \Gamma \\
& +J_{2}\left(u_{1}, \varphi\right)-J_{2}\left(u_{1}, u_{2}\right) \geq\left\langle h_{2}, \varphi-u_{2}\right\rangle, \forall \varphi \in V_{0},
\end{aligned}
$$

is turned into a fixed point problem for an operator $P:=S \circ R$ on the complete lattice $\left[\underline{u}_{1}, \bar{u}_{1}\right] \subset L^{p}(\Omega)$ which will be shown to be increasing. The operator $R$ of the composition is defined on $\left[\underline{u}_{1}, \bar{u}_{1}\right]$ by means of the variational inequality (4.2) as follows: $v_{1} \in\left[\underline{u}_{1}, \bar{u}_{1}\right], v_{1} \mapsto R v_{1}:=z$, where $z$ is the uniquely defined solution of

$$
\begin{aligned}
a^{(2)}(z, \varphi-z) & +\int_{\Gamma} g_{2}\left(\cdot, T_{2}^{0} \gamma z\right)(\gamma \varphi-\gamma z) d \Gamma \\
& +J_{2}\left(v_{1}, \varphi\right)-J_{2}\left(v_{1}, z\right) \geq\left\langle h_{2}, \varphi-z\right\rangle, \forall \varphi \in V_{0} .
\end{aligned}
$$

The existence of a unique solution of (4.3) is ensured by Lemma 3.3. Next we show that the range of $R$ is in $\left[\underline{u}_{2}, \bar{u}_{2}\right]$ and that $R$ has the following monotonicity property.

Lemma 4.2. $R:\left[\underline{u}_{1}, \bar{u}_{1}\right] \rightarrow\left[\underline{u}_{2}, \bar{u}_{2}\right]$ is decreasing (antitone), i.e., $v_{1} \leq \hat{v}_{1} \Longrightarrow$ $z \geq \hat{z}$, where $z=R v_{1}$ and $\hat{z}=R \hat{v}_{1}$.

Proof. To show $R:\left[\underline{u}_{1}, \bar{u}_{1}\right] \rightarrow\left[\underline{u}_{2}, \bar{u}_{2}\right]$, we recall the definition of $\bar{u}_{2}$ in its weak formulation (see Definition 2.1): $\bar{u}_{2} \in V$ and $\gamma \bar{u}_{2} \geq 0$ on $\partial \Omega \backslash \Gamma$ and

$$
\begin{gathered}
a^{(2)}\left(\bar{u}_{2}, \varphi\right)+\int_{\Gamma} g_{2}\left(\cdot, \gamma \bar{u}_{2}\right) \gamma \varphi d \Gamma+\int_{\Omega} f_{2}\left(\cdot, v_{1}, \bar{u}_{2}\right) \varphi d x \geq\left\langle h_{2}, \varphi\right\rangle, \\
\forall v_{1} \in\left[\underline{u}_{1}, \bar{u}_{1}\right], \forall \varphi \in V_{0} \cap L_{+}^{p}(\Omega) .
\end{gathered}
$$

Since $f_{2}\left(\cdot, v_{1}, \bar{u}_{2}\right) \in \beta_{2}\left(\cdot, v_{1}, \bar{u}_{2}\right)$ we obtain

$$
\int_{\Omega} f_{2}\left(\cdot, v_{1}, \bar{u}_{2}\right)\left(\varphi-\bar{u}_{2}\right) d x \leq J_{2}\left(v_{1}, \varphi\right)-J_{2}\left(v_{1}, \bar{u}_{2}\right), \forall \varphi \in L^{p}(\Omega),
$$

which, in particular, yields with $\varphi=\bar{u}_{2}+\left(z-\bar{u}_{2}\right)^{+}$

$$
\int_{\Omega} f_{2}\left(\cdot, v_{1}, \bar{u}_{2}\right)\left(z-\bar{u}_{2}\right)^{+} d x \leq J_{2}\left(v_{1},\left(z-\bar{u}_{2}\right)^{+}+\bar{u}_{2}\right)-J_{2}\left(v_{1}, \bar{u}_{2}\right),
$$

where $w^{+}:=\max (w, 0)$. Since $\left(z-\bar{u}_{2}\right)^{+} \in V_{0} \cap L_{+}^{p}(\Omega)$, we may take it as a special test function in (4.4), and obtain in view of (4.5) the following inequality for $\bar{u}_{2}$ :

$$
\begin{aligned}
& a^{(2)}\left(\bar{u}_{2},\left(z-\bar{u}_{2}\right)^{+}\right)+\int_{\Gamma} g_{2}\left(\cdot, \gamma \bar{u}_{2}\right) \gamma\left(z-\bar{u}_{2}\right)^{+} d \Gamma \\
& +J_{2}\left(v_{1}, \bar{u}_{2}+\left(z-\bar{u}_{2}\right)^{+}\right)-J_{2}\left(v_{1}, \bar{u}_{2}\right) \geq\left\langle h_{2},\left(z-\bar{u}_{2}\right)^{+}\right\rangle .
\end{aligned}
$$

Taking in (4.3) the special test function $\varphi=z-\left(z-\bar{u}_{2}\right)^{+}$we obtain

$$
\begin{aligned}
& \left.a^{(2)}\left(z,-\left(z-\bar{u}_{2}\right)^{+}\right)-\int_{\Gamma} g_{2}\left(\cdot, T_{2}^{0} \gamma z\right) \gamma\left(z-\bar{u}_{2}\right)^{+}\right) d \Gamma \\
& +J_{2}\left(v_{1}, z-\left(z-\bar{u}_{2}\right)^{+}\right)-J_{2}\left(v_{1}, z\right) \geq\left\langle h_{2},-\left(z-\bar{u}_{2}\right)^{+}\right\rangle .
\end{aligned}
$$


Summing up (4.6) and (4.7) gives

$$
\begin{aligned}
& a^{(2)}\left(z,\left(z-\bar{u}_{2}\right)^{+}\right)-a^{(2)}\left(\bar{u}_{2},\left(z-\bar{u}_{2}\right)^{+}\right) \\
& +\int_{\Gamma}\left(g_{2}\left(\cdot, T_{2}^{0} \gamma z\right)-g_{2}\left(\cdot, \gamma \bar{u}_{2}\right)\right) \gamma\left(z-\bar{u}_{2}\right)^{+} d \Gamma \\
& \leq J_{2}\left(v_{1}, \bar{u}_{2}+\left(z-\bar{u}_{2}\right)^{+}\right)-J_{2}\left(v_{1}, \bar{u}_{2}\right)+J_{2}\left(v_{1}, z-\left(z-\bar{u}_{2}\right)^{+}\right)-J_{2}\left(v_{1}, z\right) .
\end{aligned}
$$

By (A2) we have an estimate in the form

$$
\mu\left\|\nabla\left(z-\bar{u}_{2}\right)^{+}\right\|_{L^{p}(\Omega)}^{p} \leq a^{(2)}\left(z,\left(z-\bar{u}_{2}\right)^{+}\right)-a^{(2)}\left(\bar{u}_{2},\left(z-\bar{u}_{2}\right)^{+}\right) .
$$

The boundary integral turns out to be zero. This can easily be seen by decomposing $\Gamma$ into the two disjoint parts $\Gamma=\left\{\gamma z \geq \gamma \bar{u}_{2}\right\} \cup\left\{\gamma z<\gamma \bar{u}_{2}\right\}$, and discussing the two resulting boundary integrals separately by using $T_{2}^{0} \gamma z=$ $\gamma \bar{u}_{2}$ on $\left\{\gamma z \geq \gamma \bar{u}_{2}\right\}$, and $\gamma\left(z-\bar{u}_{2}\right)^{+}=0$ on $\left\{\gamma z<\gamma \bar{u}_{2}\right\}$. The right-hand side of (4.8) is zero, which follows in a similar way by decomposing $\Omega$ into $\Omega=\left\{z \geq \bar{u}_{2}\right\} \cup\left\{z<\bar{u}_{2}\right\}$, and discussing the individual integrals. Thus from (4.9) it follows

$$
\left\|\nabla\left(z-\bar{u}_{2}\right)^{+}\right\|_{L^{p}(\Omega)}^{p}=0
$$

which implies in view of $\left(z-\bar{u}_{2}\right)^{+} \in V_{0}$ that $\left(z-\bar{u}_{2}\right)^{+}=0$, i.e., $z \leq \bar{u}_{2}$. Analogously one can prove $\underline{u}_{2} \leq z$, which shows $R:\left[\underline{u}_{1}, \bar{u}_{1}\right] \rightarrow\left[\underline{u}_{2}, \bar{u}_{2}\right]$.

To show that $R$ is antitone, let $z=R v_{1}$ and $\hat{z}=R \hat{v}_{1}$ with $v_{1} \leq \hat{v}_{1}$. From the defining variational inequality (4.3) of $R$, we obtain by choosing the special test functions $\varphi=z+(\hat{z}-z)^{+}$and $\varphi=\hat{z}-(\hat{z}-z)^{+}$in case of the solutions $z$ and $\hat{z}$, respectively, the following inequalities:

$$
\begin{aligned}
& a^{(2)}\left(z,(\hat{z}-z)^{+}\right)+\int_{\Gamma} g_{2}\left(\cdot, T_{2}^{0} \gamma z\right) \gamma(\hat{z}-z)^{+} d \Gamma \\
& \quad+J_{2}\left(v_{1}, z+(\hat{z}-z)^{+}\right)-J_{2}\left(v_{1}, z\right) \geq\left\langle h_{2},(\hat{z}-z)^{+}\right\rangle . \\
& a^{(2)}\left(\hat{z},-(\hat{z}-z)^{+}\right)-\int_{\Gamma} g_{2}\left(\cdot, T_{2}^{0} \gamma \hat{z}\right) \gamma(\hat{z}-z)^{+} d \Gamma \\
& +J_{2}\left(\hat{v}_{1}, \hat{z}-(\hat{z}-z)^{+}\right)-J_{2}\left(\hat{v}_{1}, \hat{z}\right) \geq\left\langle h_{2},-(\hat{z}-z)^{+}\right\rangle .
\end{aligned}
$$

Summing up (4.10) and (4.11) and taking into account that $s \mapsto g_{2}(\cdot, s)$ is increasing, we obtain

$$
\begin{aligned}
& a^{(2)}\left(\hat{z},(\hat{z}-z)^{+}\right)-a^{(2)}\left(z,(\hat{z}-z)^{+}\right) \\
& \leq J_{2}\left(v_{1}, z+(\hat{z}-z)^{+}\right)-J_{2}\left(v_{1}, z\right)+J_{2}\left(\hat{v}_{1}, \hat{z}-(\hat{z}-z)^{+}\right)-J_{2}\left(\hat{v}_{1}, \hat{z}\right) .
\end{aligned}
$$

We discuss the right-hand side of (4.12) by using the definition of the functional $J_{2}$ given in (3.2) and by employing the monotonicity property of $f_{2}$ in the competing species case, as well as the decomposition of $\Omega=\{\hat{z} \geq z\} \cup\{\hat{z}<z\}$. 
Let us denote the right-hand side of (4.12) by $J$. Then it can easily be seen that the integral contribution is zero for $x \in\{\hat{z}<z\}$, and thus

$$
\begin{aligned}
J & =\int_{\{\hat{z} \geq z\}}\left(\int_{0}^{\hat{z}(x)} f_{2}^{\alpha}\left(x, v_{1}(x), s\right)-\int_{0}^{z(x)} f_{2}^{\alpha}\left(x, v_{1}(x), s\right) d s\right) d x \\
& +\int_{\{\hat{z} \geq z\}}\left(\int_{0}^{z(x)} f_{2}^{\alpha}\left(x, \hat{v}_{1}(x), s\right)-\int_{0}^{\hat{z}(x)} f_{2}^{\alpha}\left(x, \hat{v}_{1}(x), s\right) d s\right) d x \\
& =\int_{\{\hat{z} \geq z\}}\left(\int_{z(x)}^{z(x)}\left(f_{2}^{\alpha}\left(x, v_{1}(x), s\right)-f_{2}^{\alpha}\left(x, \hat{v}_{1}(x), s\right)\right) d s\right) d x \\
& \leq 0 .
\end{aligned}
$$

By (A2) we obtain in view of (4.12), (4.13)

$$
\mu\left\|\nabla(\hat{z}-z)^{+}\right\|_{L^{p}(\Omega)}^{p} \leq a^{(2)}\left(\hat{z},(\hat{z}-z)^{+}\right)-a^{(2)}\left(z,(\hat{z}-z)^{+}\right) \leq 0,
$$

which implies $(\hat{z}-z)^{+}=0$, and thus $\hat{z} \leq z$, i.e., $R$ is antitone or decreasing.

By means of (4.1) we define the operator $S$ in the composition $P=S \circ R$ on the range of $R$ as follows: $z=R v_{1} \in\left[\underline{u}_{2}, \bar{u}_{2}\right], z \mapsto S z=u_{1}$, where $u_{1}$ is the uniquely defined solution of the variational inequality:

$$
\begin{aligned}
a^{(1)}\left(u_{1}, \varphi-u_{1}\right) & +\int_{\Gamma} g_{1}\left(\cdot, T_{1}^{0} \gamma u_{1}\right)\left(\gamma \varphi-\gamma u_{1}\right) d \Gamma \\
& +J_{1}(\varphi, z)-J_{1}\left(u_{1}, z\right) \geq\left\langle h_{1}, \varphi-u_{1}\right\rangle, \forall \varphi \in V_{0} .
\end{aligned}
$$

The following result for the operator $S$ can be proved in just the same way as Lemma 4.2.

Lemma 4.3. $S: R\left(\left[\underline{u}_{1}, \bar{u}_{1}\right]\right) \subset\left[\underline{u}_{2}, \bar{u}_{2}\right] \rightarrow\left[\underline{u}_{1}, \bar{u}_{1}\right]$ is decreasing (antitone), i.e., $z \leq \hat{z} \Longrightarrow u_{1} \geq \hat{u}_{1}$, where $u_{1}=S z$ and $\hat{u}_{1}=S \hat{z}$.

To complete the existence proof of Theorem 4.1 we note that by Lemma 4.2 and Lemma 4.3 the operator $P=S \circ R:\left[\underline{u}_{1}, \bar{u}_{1}\right] \rightarrow\left[\underline{u}_{1}, \bar{u}_{1}\right]$ is well-defined and, as the composition of two decreasing operators, $P$ is increasing. Since $\left[\underline{u}_{1}, \bar{u}_{1}\right] \subset L^{p}(\Omega)$ is a complete lattice, we may apply Lemma 4.1 (Tarski's fixed point theorem), which asserts the existence of greatest and smallest fixed points of $P$. Let $u_{1}$ be any fixed point of $P$, i.e., $u_{1}=P u_{1}=S\left(R u_{1}\right)$, and denote $u_{2}=R u_{1}$, then one readily verifies that the pair $\left(u_{1}, u_{2}\right) \in[\underline{u}, \bar{u}]$ is a solution of the coupled system (3.5) of variational inequalities, and thus of the system (2.11), which completes the proof of Theorem 4.1 .

Corollary 4.1. Let the cartesian product $L^{p}(\Omega) \times L^{p}(\Omega)$ be equipped with the partial ordering $\preceq$, defined by

$$
\left(u_{1}, u_{2}\right) \preceq\left(w_{1}, w_{2}\right) \Longleftrightarrow u_{1} \leq w_{1} \wedge u_{2} \geq w_{2},
$$


where the order for the components is the usual natural partial ordering in $L^{p}(\Omega)$ introduced in section 2. Then under the hypotheses of Theorem 4.1 there exist a greatest and a smallest solution of the multivalued system (2.11) within the trapping region $[\underline{u}, \bar{u}]$ with respect to the ordering $\preceq$.

Proof. First one readily observes that $u=\left(u_{1}, u_{2}\right)$ is a solution of (2.11) if and only if it defines a fixed point of the operator $P=S \circ R$. Let $u_{1}^{*}$ and $u_{1, *}$ denote the greatest and smallest fixed point of $P$ in $\left[\underline{u}_{1}, \bar{u}_{1}\right]$, respectively, obtained by Tarski's fixed point theorem. Define $u_{2}^{*}:=R u_{1}^{*}$ and $u_{2, *}:=R u_{1, *}$. Then for any solution $u=\left(u_{1}, u_{2}\right)$ one easily gets $u_{*} \preceq u \preceq u^{*}$ with $u_{*}=\left(u_{1, *}, u_{2, *}\right)$ and $u^{*}=\left(u_{1}^{*}, u_{2}^{*}\right)$.

Denote by $\mathcal{S}$ the set of all solutions of (2.11) within the trapping region. Then under additional continuity assumptions of the components $f_{k}$ in their nonprincipal arguments we show the following compactness result.

Corollary 4.2. Let the hypotheses of Theorem 4.1 be satisfied, and assume, in addition, to hypothesis (H2) that each $f_{k}, k=1,2$, is continuous in its nonprincipal argument uniformly with respect to its principal argument, i.e., $s_{j} \mapsto f_{k}\left(\cdot, s_{1}, s_{2}\right)$ is continuous for $j=1,2$, with $j \neq k$ uniformly in $s_{k}$. Then $\mathcal{S}$ is compact in $V_{0} \times V_{0}$.

Proof. Any element $u \in \mathcal{S}$ satisfies the system (3.5) of variational inequalities written in the form

$$
\begin{aligned}
& \left\langle A_{1} u_{1}, \varphi-u_{1}\right\rangle+\left\langle G_{1} u_{1}, \varphi-u_{1}\right\rangle \\
& +J_{1}\left(\varphi, u_{2}\right)-J_{1}\left(u_{1}, u_{2}\right) \geq\left\langle h_{1}, \varphi-u_{1}\right\rangle, \forall \varphi \in V_{0}, \\
& \left\langle A_{2} u_{2}, \varphi-u_{2}\right\rangle+\left\langle G_{2} u_{2}, \varphi-u_{2}\right\rangle \\
& +J_{2}\left(u_{1}, \varphi\right)-J_{2}\left(u_{1}, u_{2}\right) \geq\left\langle h_{2}, \varphi-u_{2}\right\rangle, \forall \varphi \in V_{0},
\end{aligned}
$$

where for $k=1,2$,

$$
\left\langle G_{k} u_{k}, \varphi\right\rangle=\int_{\Gamma} g_{k}\left(\cdot, T_{k}^{0} \gamma u_{k}\right) \gamma \varphi d \Gamma, \quad \forall \varphi \in V_{0}
$$

We show first that the functionals $J_{k}:[\underline{u}, \bar{u}] \subset L^{p}(\Omega) \times L^{p}(\Omega) \rightarrow \mathbb{R}, k=1,2$, are continuous, i.e., $J_{k}\left(u_{1, n}, u_{2, n}\right) \rightarrow J_{k}\left(u_{1}, u_{2}\right)$ whenever $\left(u_{1, n}, u_{2, n}\right) \rightarrow\left(u_{1}, u_{2}\right)$ in $L^{p}(\Omega) \times L^{p}(\Omega)$. Let $k=1$. By Lemma 3.1 we already know that $J_{1}\left(\cdot, u_{2}\right)$ : $\left[\underline{u}_{1}, \bar{u}_{1}\right] \rightarrow \mathbb{R}$ is uniformly Lipschitz continuous in its principal argument. Thus we only need to show that $J_{1}\left(u_{1}, \cdot\right):\left[\underline{u}_{2}, \bar{u}_{2}\right] \rightarrow \mathbb{R}$ is continuous for fixed principal argument $u_{1}$. This latter, however, follows from the continuity assumption 
of $f_{1}\left(\cdot, s_{1}, s_{2}\right)$ in its nonprincipal argument $s_{2}$ as follows.

$$
\begin{aligned}
& \left|J_{1}\left(u_{1}, u_{2, n}\right)-J_{1}\left(u_{1}, u_{2}\right)\right| \\
& =\mid \int_{\Omega}\left(\int_{0}^{u_{1}(x)}\left(f_{1}^{\alpha}\left(x, s, u_{2, n}(x)\right)-f_{1}^{\alpha}\left(x, s, u_{2}(x)\right)\right) d s\right) d x, \\
& \leq\left(\int_{0}^{1}\left\|F_{1}^{\alpha}\left(s(t), u_{2, n}\right)-F_{1}^{\alpha}\left(s(t), u_{2}\right)\right\|_{L^{q}(\Omega)} d t\right)\left\|u_{1}\right\|_{L^{p}(\Omega)},
\end{aligned}
$$

where $F_{1}^{\alpha}$ denotes the Nemytskij operator associated with $f_{1}^{\alpha}$, and where we have used the substitution $s(t)(x):=t u_{1}(x), t \in[0,1]$. Since the Nemytskij operator $F_{1}^{\alpha}(s(t), \cdot): L^{p}(\Omega) \rightarrow L^{q}(\Omega)$ is continuous uniformly in its principal argument, the right-hand side of (4.17) tends to zero as $n \rightarrow \infty$, and thus the continuity of $J_{1}:[\underline{u}, \bar{u}] \subset L^{p}(\Omega) \times L^{p}(\Omega) \rightarrow \mathbb{R}$. Similarly, one proves the continuity of $J_{2}$. Let $\left(u_{1, n}, u_{2, n}\right) \in \mathcal{S}$ be any sequence. Then this sequence is bounded in $L^{p}(\Omega) \times L^{p}(\Omega)$, which implies that $\left|J_{k}\left(u_{1, n}, u_{2, n}\right)\right| \leq c$ for all $n$. Since $\left(u_{1, n}, u_{2, n}\right)$ satisfy (4.15) and (4.16), we obtain from (4.15) with $\varphi=0$ :

$$
\begin{aligned}
& \left\langle A_{1} u_{1, n},-u_{1, n}\right\rangle+\left\langle G_{1} u_{1, n},-u_{1, n}\right\rangle \\
& +J_{1}\left(0, u_{2, n}\right)-J_{1}\left(u_{1, n}, u_{2, n}\right) \geq\left\langle h_{1},-u_{1, n}\right\rangle, \forall n,
\end{aligned}
$$

which yields

$$
\begin{aligned}
& \left\langle A_{1} u_{1, n}, u_{1, n}\right\rangle+\left\langle G_{1} u_{1, n}, u_{1, n}\right\rangle \\
& \leq\left|J_{1}\left(0, u_{2, n}\right)\right|+\left|J_{1}\left(u_{1, n}, u_{2, n}\right)\right|+\left\|h_{1}\right\|_{V_{0}^{*}}\left\|u_{1, n}\right\|_{V_{0}} .
\end{aligned}
$$

By the coercivity of the operator $A_{1}+G_{1}: V_{0} \rightarrow V_{0}^{*}$ and the uniform boundedness of the functional $J_{1}$ we obtain from (4.19) that $\left\|u_{1, n}\right\|_{V_{0}} \leq c, \quad \forall n$. Analogously, one shows $\left\|u_{2, n}\right\|_{V_{0}} \leq c, \forall n$. Thus there is a subsequence, again denoted by $\left(u_{1, n}, u_{2, n}\right)$, which is weakly convergent in $V_{0} \times V_{0}$ to $\left(u_{1}, u_{2}\right)$. We shall show that this subsequence is strongly convergent in $V_{0} \times V_{0}$ to $\left(u_{1}, u_{2}\right)$, and that the limit $\left(u_{1}, u_{2}\right) \in \mathcal{S}$. Consider (4.15) satisfied by $u_{1, n}$ and $u_{2, n}$, and use the special test function $\varphi=u_{1}$ to obtain

$$
\begin{aligned}
\mu \| \nabla\left(u_{1}-u_{1, n} \|_{L^{p}(\Omega)}^{p}\right. & \leq\left\langle A_{1} u_{1}-A_{1} u_{1, n}, u_{1}-u_{1, n}\right\rangle \\
& \leq\left\langle A_{1} u_{1}, u_{1}-u_{1, n}\right\rangle+\left\langle G_{1} u_{1, n}, u_{1}-u_{1, n}\right\rangle \\
& +J_{1}\left(u_{1}, u_{2, n}\right)-J_{1}\left(u_{1, n}, u_{2, n}\right)+\left\langle h_{1}, u_{1, n}-u_{1}\right\rangle .
\end{aligned}
$$

Using the weak convergence of $u_{1, n} \rightarrow u_{1}$ in $V_{0}$, the compact embedding $V_{0} \subset L^{p}(\Omega)$, the compactness of the trace operator $\gamma: V \rightarrow L^{p}(\partial \Omega)$, and the continuity of the functional $J_{1}$, the right-hand side of (4.20) tends to zero as $n \rightarrow \infty$. Thus, from (4.20) it follows that $\left(u_{1, n}\right)$ is strongly convergent in $V_{0}$ to $u_{1}$. Analogously also $\left(u_{2, n}\right)$ is strongly convergent in $V_{0}$ to $u_{2}$. The strong convergence of the solutions $\left(u_{1, n}, u_{2, n}\right) \in V_{0} \times V_{0}$ satisfying the system (4.15) and (4.16) of variational inequalities, the continuity of the operator $A_{k}+G_{k}: V_{0} \rightarrow V_{0}^{*}$, and the continuity of the functionals $J_{k}$ allow passage to the limit as $n \rightarrow \infty$, which also shows that the limit $\left(u_{1}, u_{2}\right)$ belongs to $\mathcal{S}$ and thus the assertion is proved. 
Following the ideas developed so far, we can also prove a result analogous to Theorem 4.1 for the model of cooperating species.

Theorem 4.2. Let $f=\left(f_{1}, f_{2}\right)$ be the vector field corresponding to the cooperating species model. Assume (A1) and (A2), and let $[\underline{u}, \bar{u}] \subset V \times V$ be a trapping region for the $B V P$ (1.1) according to Definition 2.1. Let $\alpha>$ 0 be any given constant so that (2.1) of (H1) is satisfied with respect to $[\underline{u}-(\alpha, \alpha), \bar{u}+(\alpha, \alpha)]$, and (H2) with respect to $\left[\underline{u}_{k}, \bar{u}_{k}\right], k=1,2$, where $\underline{u}_{k}$ and $\bar{u}_{k}$ are the components of $\underline{u}$ and $\bar{u}$ of the trapping interval. Then there exist solutions of the system (2.11) within $[\underline{u}, \bar{u}]$.

Proof. The proof can be carried out similarly to that of Theorem 4.1, and is again based on the system of variational inequalities (3.5) and on Tarski's fixed point theorem. The only difference in the proof comes from the opposite monotone behavior of the operators $R$ and $S$ of the fixed point operator $P=$ $S \circ R$. Define $R$ and $S$ in just the same way as in the proof of Theorem 4.1. Then $R:\left[\underline{u}_{1}, \bar{u}_{1}\right] \rightarrow\left[\underline{u}_{2}, \bar{u}_{2}\right]$ is increasing, and $S: R\left(\left[\underline{u}_{1}, \bar{u}_{1}\right]\right) \subset\left[\underline{u}_{2}, \bar{u}_{2}\right] \rightarrow\left[\underline{u}_{1}, \bar{u}_{1}\right]$ is increasing as well, such that $P:\left[\underline{u}_{1}, \bar{u}_{1}\right] \rightarrow\left[\underline{u}_{1}, \bar{u}_{1}\right]$ is again an increasing operator, which yields the assertion of the Theorem. So let us only demonstrate that $R:\left[\underline{u}_{1}, \bar{u}_{1}\right] \rightarrow\left[\underline{u}_{2}, \bar{u}_{2}\right]$ is increasing. To this end, let $z=R v_{1}$ and $\hat{z}=R \hat{v}_{1}$ with $v_{1} \leq \hat{v}_{1}$. From the defining variational inequality (4.3) of $R$ we obtain by choosing the special test functions $\varphi=z-(z-\hat{z})^{+}$and $\varphi=\hat{z}+(z-\hat{z})^{+}$in case of the solution $z$ and $\hat{z}$, respectively, the following inequalities:

$$
\begin{aligned}
& a^{(2)}\left(z,-(z-\hat{z})^{+}\right)-\int_{\Gamma} g_{2}\left(\cdot, T_{2}^{0} \gamma z\right) \gamma(z-\hat{z})^{+} d \Gamma \\
& +J_{2}\left(v_{1}, z-(z-\hat{z})^{+}\right)-J_{2}\left(v_{1}, z\right) \geq\left\langle h_{2},-(z-\hat{z})^{+}\right\rangle . \\
& \quad a^{(2)}\left(\hat{z},(z-\hat{z})^{+}\right)+\int_{\Gamma} g_{2}\left(\cdot, T_{2}^{0} \gamma \hat{z}\right) \gamma(z-\hat{z})^{+} d \Gamma \\
& \quad+J_{2}\left(\hat{v}_{1}, \hat{z}+(z-\hat{z})^{+}\right)-J_{2}\left(\hat{v}_{1}, \hat{z}\right) \geq\left\langle h_{2},(z-\hat{z})^{+}\right\rangle .
\end{aligned}
$$

Summing (4.21) and (4.22) and taking into account that $s \mapsto g_{2}(\cdot, s)$ is increasing we obtain

$$
\begin{aligned}
& a^{(2)}\left(z,(z-\hat{z})^{+}\right)-a^{(2)}\left(\hat{z},(z-\hat{z})^{+}\right) \\
& \leq J_{2}\left(v_{1}, z-(z-\hat{z})^{+}\right)-J_{2}\left(v_{1}, z\right)+J_{2}\left(\hat{v}_{1}, \hat{z}+(z-\hat{z})^{+}\right)-J_{2}\left(\hat{v}_{1}, \hat{z}\right) .
\end{aligned}
$$

We discuss the right-hand side of (4.23) by using the definition of the functional $J_{2}$ given in (3.2) and by employing the monotonicity property of $f_{2}$ in the cooperating species case, as well as the decomposition: $\Omega=\{z \geq \hat{z}\} \cup\{z<\hat{z}\}$. Let us denote the right-hand side of (4.23) by $J$. Then it can easily be seen 
that the integral contribution is zero in $\{z<\hat{z}\}$, and thus

$$
\begin{aligned}
J & =\int_{\{z \geq \hat{z}\}}\left(\int_{0}^{\hat{z}(x)} f_{2}^{\alpha}\left(x, v_{1}(x), s\right)-\int_{0}^{z(x)} f_{2}^{\alpha}\left(x, v_{1}(x), s\right) d s\right) d x \\
& +\int_{\{z \geq \hat{z}\}}\left(\int_{0}^{z(x)} f_{2}^{\alpha}\left(x, \hat{v}_{1}(x), s\right)-\int_{0}^{\hat{z}(x)} f_{2}^{\alpha}\left(x, \hat{v}_{1}(x), s\right) d s\right) d x \\
& =\int_{\{z \geq \hat{z}\}}\left(\int_{z(x)}^{z(x)}\left(f_{2}^{\alpha}\left(x, v_{1}(x), s\right)-f_{2}^{\alpha}\left(x, \hat{v}_{1}(x), s\right)\right) d s\right) d x \\
& =\int_{\{z \geq \hat{z}\}}\left(\int_{\hat{z}(x)}^{z(x)}\left(f_{2}^{\alpha}\left(x, \hat{v}_{1}(x), s\right)-f_{2}^{\alpha}\left(x, v_{1}(x), s\right)\right) d s\right) d x \\
& \leq 0 .
\end{aligned}
$$

By (A2),(4.23) and (4.24) we get

$$
\mu\left\|\nabla(z-\hat{z})^{+}\right\|_{L^{p}(\Omega)}^{p} \leq a^{(2)}\left(z,(z-\hat{z})^{+}\right)-a^{(2)}\left(\hat{z},(z-\hat{z})^{+}\right) \leq 0,
$$

which implies $(z-\hat{z})^{+}=0$, and thus $z \leq \hat{z}$, i.e., $R$ is increasing.

For completeness we formulate the extremality and compactness result that hold for cooperating species.

Corollary 4.3. Let the cartesian product $L^{p}(\Omega) \times L^{p}(\Omega)$ be equipped with the natural partial ordering $\leq$, i.e.,

$$
\left(u_{1}, u_{2}\right) \leq\left(w_{1}, w_{2}\right) \Longleftrightarrow u_{1} \leq w_{1} \wedge u_{2} \leq w_{2} .
$$

Then under the hypotheses of Theorem 4.2 there exist a greatest and a smallest solution of the multivalued system (2.11) within the trapping region $[\underline{u}, \bar{u}]$. Moreover, under the additional continuity assumption of Corollary 4.2, the solution set $\mathcal{S}$ is compact in $V_{0} \times V_{0}$.

The proof of Corollary 4.3 follows immediately from that of Corollary 4.1 and Corollary 4.2 .

\section{Applications}

In this section, we shall provide two applications of the theory developed in the paper. We begin with a mixed monotone system of cooperative species.

\subsection{Cooperative Species}

We consider the steady-state transport of two species of opposite charge within

a physical channel, such as occurs in chemistry or biology. We assume that a 
specified potential $u(x)$ is maintained within the channel. This is equivalent to the statement that the permanent charge of the channel is much larger than the net mobile charge. We are interested in the case of 'partial equilibration' (pumping), in which a virtual source switches on when the concentration of one species becomes too large in relation to the other. The model thus merges the source-free transport of ions with the episodic action of ion pumps, which restore the levels of ion species in the baths adjoining the channels, so as to obtain a steady qualitative model.

If the concentrations of the anion and cation species are denoted by $n$ and $p$, we have the equations:

$$
\begin{aligned}
\nabla \cdot\left[D_{n} \nabla n-\mu_{n} n \nabla u\right]+S_{n} & =0, \\
-\nabla \cdot\left[D_{p} \nabla p+\mu_{p} p \nabla u\right]-S_{p} & =0 .
\end{aligned}
$$

Here, $-\nabla u$ is the electric (force) field. Each equation describes the divergence of the respective charge density in a conservation statement. Also, $\mu_{n}, \mu_{p}$ denote mobility coefficients, $D_{n}, D_{p}$ denote diffusion coefficients, and $S_{n}, S_{p}$ denote virtual source terms, which we elaborate below. If new variables, $v, w$ (so-called quasi-Fermi levels), are introduced so that $n=\exp (u-v)$ and $p=\exp (w-u)$, then, under the assumption of Einstein's relations, as well as the choice of units in which the thermal voltage is unity, the system (5.1) and (5.2) is rewritten as

$$
\begin{aligned}
& -\nabla \cdot\left[\mu_{n} e^{u-v} \nabla v\right]+S_{n}=0 \\
& -\nabla \cdot\left[\mu_{p} e^{w-u} \nabla w\right]-S_{p}=0 .
\end{aligned}
$$

The boundary conditions for the problem are Dirichlet boundary conditions of so-called charge neutral type, for which $v=u, w=u$.

If a third set of variables is introduced, the Slotboom variables, $V=\exp (-v)$, $W=\exp (w)$, and the mobility coefficients are assumed constant, then the system reduces to the linear format in the variables $V, W$,

$$
\begin{array}{r}
-\nabla \cdot\left[e^{u} \nabla V\right]-S_{n}=0, \\
-\nabla \cdot\left[e^{-u} \nabla W\right]-S_{p}=0,
\end{array}
$$

where we have absorbed the mobility coefficients in the source terms.

We propose virtual sources of the following form. First, we give a verbal description of $S_{n}$. If $n$ is less than $p$ by more than a given function of $x$, say $\delta_{1}(x)$, then the source $S_{n}$ is 'switched on', with a position dependent strength, allowing for geometric effects; the sign is, of course, positive. The interpretation is that the transport of $n$ is equilibrated. Otherwise, the virtual source is 
zero. The situation for $S_{p}$ is 'mirror reversed'. In equation form, we write:

$$
\begin{aligned}
& S_{n}(x, V, W)=\left\{\begin{array}{l}
c_{1}(x), \text { if } \delta_{1}(x)<W \exp (-u(x))-V \exp (u(x)), \\
0, \text { otherwise. }
\end{array}\right. \\
& S_{p}(x, V, W)=\left\{\begin{array}{l}
c_{2}(x), \text { if } \delta_{2}(x)<V \exp (u(x))-W \exp (-u(x)), \\
0, \text { otherwise. }
\end{array}\right.
\end{aligned}
$$

One sees that $-S_{n}$ is increasing in $V$ for each fixed $x, W$; and decreasing in $W$ for each fixed $x, V$. Similarly, $-S_{p}$ is increasing in $W$ for each fixed $x, V$; and decreasing in $V$ for each fixed $x, W$. The system is thus an example of cooperative species, and is of mixed monotonicity. The least regularity assumption for the potential $u$ is $u \in W^{2,2}(\Omega)$; this implies, by the Sobolev Embedding Theorem, $u \in C(\bar{\Omega})$ provided that $2>N / 2$. The measurability assumption of hypothesis (H1) on the nonlinearities $S_{n}$ and $S_{p}$ which have jump discontinuities in the variables $V$ and $W$ are satisfied if the functions $c_{k}, \delta_{k}: \Omega \rightarrow \mathbb{R}$, $k=1,2$, are not 'too bad', e.g., Borel measurable, cf. [1].

The system is not quite in the form of the theory of (1.1). In terms of the Slotboom variables, we have the boundary conditions: $\Gamma=\emptyset, V=\exp (-u), W=$ $\exp (u)$, on $\partial \Omega$. Thus, we make the final change of variable,

$$
Y=V-\exp (-u), Z=W-\exp (u)
$$

The system is transformed in the typical way to:

$$
\begin{gathered}
-\nabla \cdot\left[e^{u} \nabla Y\right]-\tilde{S}_{n}=-\Delta u, \\
-\nabla \cdot\left[e^{-u} \nabla Z\right]-\tilde{S}_{p}=\Delta u .
\end{gathered}
$$

Here, $\tilde{S}_{n}, \tilde{S}_{p}$ act on $Y, Z$ via the action of $S_{n}, S_{p}$ on the corresponding $V, W$. Note that $Y, Z$ are required to have zero boundary trace. In order to determine the interval which defines a trapping region in $Y \times Z$ space, we begin by introducing the auxiliary functions $\underline{\mathrm{V}}$ and $\bar{V}$ via the solutions of the Poisson equations:

$$
\begin{gathered}
-\nabla(\exp (u) \nabla \underline{\mathrm{V}})=0, \quad \underline{\mathrm{V}}=\exp (-u) \text { on } \partial \Omega, \\
-\nabla(\exp (u) \nabla \bar{V})=c_{1}(x), \quad \bar{V}=\exp (-u) \text { on } \partial \Omega .
\end{gathered}
$$

We then define: $\underline{Y}=\underline{\mathrm{V}}-\exp (-u)$ and $\bar{Y}=\bar{V}-\exp (-u)$. Similarly, we introduce the auxiliary functions $\underline{\mathrm{W}}$ and $\bar{W}$ :

$$
\begin{gathered}
-\nabla(\exp (-u) \nabla \underline{\mathrm{W}})=0, \quad \underline{\mathrm{W}}=\exp (u) \text { on } \partial \Omega, \\
-\nabla(\exp (-u) \nabla \bar{W})=c_{2}(x), \quad \bar{V}=\exp (-u) \text { on } \partial \Omega .
\end{gathered}
$$


We then define: $\underline{\mathrm{Z}}=\underline{\mathrm{W}}-\exp (u)$ and $\bar{Z}=\bar{W}-\exp (u)$. The interval,

$$
[\underline{\mathrm{Y}}, \bar{Y}] \times[\underline{\mathrm{Z}}, \bar{Z}]
$$

is now seen to be a trapping region. One deduces obvious bounds for $n, p$.

\subsection{Competitive Species}

We consider a fluid medium which may undergo a change of phase, and which acts as a carrier for a certain solute species. Included would be the case of a solute dissolved in water, such that the resulting solvent may undergo freezing. It is known that such impurities can affect thermal properties, such as the temperature at which freezing occurs. The example we study is a typical semidiscrete elliptic system, obtained by applying the method of horizontal lines to the parabolic system. We will use the enthalpy transformation, and, with our theory, introduce an enthalpy which depends both upon temperature and the concentration of the solute. This appears to be the first time such a general two-phase Stefan problem has been considered.

If $k_{0}$ is the thermal conductivity, depending upon temperature $\theta$ and solute concentration $v$, and the Kirchhoff transformation,

$$
u=K \circ \theta=\int_{0}^{\theta} k_{0}(\xi, v) d \xi
$$

is employed, one obtains a generalized temperature $u$ (see [7, Section 1.1]). In our case, we think of the transformation employed for each fixed solute distribution $v$. We designate by $Q$ the standard enthalpy, typically consisting of the sum of a regular term, obtained by an integration of the volumetric heat capacity, and a discontinuous term, the so-called latent energy content. The latter term is discontinuous at the nominal change of phase temperature. We thus have the following parabolic distribution system, where we write $H=Q \circ K^{-1}$.

$$
\frac{\partial H(u, v)}{\partial t}-\Delta u=0, \quad \frac{\partial v}{\partial t}-\Delta v+G(u)=0 .
$$

The two dependent variables are the generalized temperature $u$ and the solute concentration $v$, where standard diffusion is assumed. If we think of $-G$ as a (positive) control source function for the solute, with less solute required at higher temperatures, then $G$ is increasing with $u$, and may be discontinuous. We do assume, however, that $G$ is bounded, and set $C=\sup _{s}|G(s)|$. $H$ is assumed increasing in $u, v$, and is expected to be discontinuous in $u$ for each fixed $v$. If a fully implicit semidiscretization is employed, we have, formally,

$$
H\left(u_{k}, v_{k}\right) / \Delta t-\Delta u_{k}=H\left(u_{k-1}, v_{k-1}\right) / \Delta t .
$$




$$
v_{k} / \Delta t-\Delta v_{k}+G\left(u_{k}\right)=v_{k-1} / \Delta t .
$$

Here, $t_{k}=k \Delta t$ within a uniform grid spacing, and $u_{k}, v_{k}$ may be viewed as approximations at time $t_{k}$. We shall select arbitrary linear Robin boundary conditions on $\Gamma$, i.e., we assume $\partial u / \partial \nu+b_{u} u=0$ and $\partial v / \partial \nu+b_{v} v=0$, where $b_{u}, b_{v}$ are positive constants. We choose a nonegative temperature scale (say, Kelvin), and a small grounded region $\partial \Omega \backslash \Gamma$ on the boundary. Thus the semidiscrete system (5.12) and (5.13) is an example of a competitive species system.

In order to obtain a trapping region for the latter let us assume that the initial values for $u$ and $v$, i.e., $u(x, 0)=u_{0}(x)$ and $v(x, 0)=v_{0}(x)$ are nonnegative, measurable, and bounded functions. Since $-G$ is positive and $G$ is assumed to be bounded with $C=\sup _{s}|G(s)|$, one gets uniform bounds for $v$ from (5.11) by means of the maximum principle in the form

$$
0 \leq v(x, t) \leq\left\|v_{0}\right\|_{\infty}+C t .
$$

Let $\left[0, t_{0}\right]$ be the given time interval. In order to get the interval for the variable $v$ of the semidiscrete system (5.12) and (5.13), it is enough to find $\underline{v}$ and $\bar{v}$, which are lower and upper solutions, respectively, of

$$
\begin{gathered}
\underline{v} / \Delta t-\Delta \underline{v}=v_{k-1} / \Delta t, \quad \frac{\partial \underline{v}}{\partial \nu}+b_{v} \underline{v}=0 \quad \text { on } \Gamma, \underline{v}=0 \quad \text { on } \partial \Omega \backslash \Gamma, \\
\bar{v} / \Delta t-\Delta \bar{v}=C+v_{k-1} / \Delta t, \quad \frac{\partial \bar{v}}{\partial \nu}+b_{v} \bar{v}=0 \quad \text { on } \Gamma, \bar{v}=0 \quad \text { on } \partial \Omega \backslash \Gamma .
\end{gathered}
$$

This can be obtained independently of $k$ by using (5.14), and one verifies that $\underline{v}=0$ and $\bar{v}=\left\|v_{0}\right\|_{\infty}+t_{0} C$ are such uniform lower and upper solutions. By using these uniform bounds for $v$ we may now define the interval in the variable $u$ for the semidiscrete system. On the horizontal line $t=t_{k}$ lower and upper bounds for $u$ are given by the unique solutions $\underline{u}$ and $\bar{u}$, respectively, to the Robin-Dirichlet boundary value problems,

$$
\begin{aligned}
& \frac{H(\underline{u}, \bar{v})}{\Delta t}-\Delta \underline{u}=\frac{H\left(u_{k-1}, \underline{v}\right)}{\Delta t}, \quad \frac{\partial \underline{u}}{\partial \nu}+b_{u} \underline{u}=0 \quad \text { on } \Gamma, \underline{u}=0 \quad \text { on } \partial \Omega \backslash \Gamma, \\
& \frac{H(\bar{u}, \underline{v})}{\Delta t}-\Delta \bar{u}=\frac{H\left(u_{k-1}, \bar{v}\right)}{\Delta t}, \quad \frac{\partial \bar{u}}{\partial \nu}+b_{u} \bar{u}=0 \quad \text { on } \Gamma, \bar{u}=0 \quad \text { on } \partial \Omega \backslash \Gamma,
\end{aligned}
$$

where $\underline{v}=0$ and $\bar{v}=\left\|v_{0}\right\|_{\infty}+t_{0} C$. One also readily verifies that any lower solution of (5.15) and any upper solution of (5.16) are lower and upper bounds for $u$. If we make use of the assumed monotonicity properties of $H$, we see that $[\underline{u}, \bar{u}] \times[\underline{v}, \bar{v}]$ forms a trapping region for the semidiscrete problem (5.12), (5.13). 


\section{References}

[1] J. Appell and P. P. Zabrejko, Nonlinear Superposition Operators, Cambridge University Press, New York, 1990.

[2] S. Carl and C. Grossmann, Monotone enclosure for elliptic and parabolic systems with nonmonotone nonlinearities, J. Math. Anal. Appl. 151 (1990), 190-202.

[3] S. Carl and S. Heikkilä, Nonlinear Differential Equations in Ordered Spaces, CRC Press, 2000.

[4] S. Carl, S. Heikkilä, and M. Kumpulainen, On a generalized iteration method with applications to fixed point theorems and elliptic systems involving discontinuities, Nonlinear Anal. 20 (1993), 157-167.

[5] K.C. Chang, Variational methods for non-differentiable functionals and their applications to partial differential equations, J. Math. Anal. Appl. 80 (1981), $102-129$.

[6] J. Heinonen, T. Kilpeläinen, and O. Martio, Nonlinear Potential Theory of Degenerate Elliptic Equations, Clarendon Press, Oxford, 1993.

[7] J. W. Jerome, Approximation of Nonlinear Evolution Systems, Academic Press, 1983.

[8] J. W. Jerome, A trapping principle for discontinuous elliptic systems of mixed monotone type, J. Math. Anal. Appl. (to appear).

[9] A. Kufner, O. John, and S. Fucik,Function Spaces, Nordhoff International Publishing, Leyden, 1977.

[10] G. S. Ladde, V. Lakshmikantham, and A. S. Vatsala, Monotone Iterative Techniques for Nonlinear Differential Equations, Pitman, Boston, 1985.

[11] E. Mitidieri and G. Sweers, Existence of a maximal solution for quasimonotone elliptic systems, Differential Integral Equations 7 (1994), 1495-1510.

[12] E. Zeidler, Nonlinear Functional Analysis and its Applications, Vol. I: FixedPoint Theorems, Springer-Verlag, Berlin, 1985.

[13] E. Zeidler, Nonlinear Functional Analysis and its Applications, Vol. III: Variational Methods, Springer-Verlag, Berlin, 1985. 